\title{
Correlation of Etch Pits and Dislocations in As-grown and Thermal-Cycle-Annealed HgCdTe(211) Films
}

\author{
Majid. Vaghayenegar ${ }^{1}$, R. N. Jacobs ${ }^{2}$, J. D. Benson ${ }^{2}$, A. J. Stoltz ${ }^{2}$, L. A. Almeida ${ }^{2}$, and David. J. Smith ${ }^{3}$ \\ 1. School for Engineering of Matter, Transport and Energy, Arizona State University, Tempe, AZ 85287 \\ 2. U.S. Army RDECOM, CERDEC Night Vision and Electronic Sensors Directorate, Fort Belvoir, VA \\ 22060 \\ 3. Department of Physics, Arizona State University, Tempe, AZ 85287
}

$\mathrm{Hg}_{\mathrm{x}} \mathrm{Cd}_{1-\mathrm{x}} \mathrm{Te}$ (MCT), has been the primary material used for infrared (IR) detectors and sensors [1]. Serious limitations such as larger initial defect density, high price $\left(400 \$ / \mathrm{cm}^{2}\right)$, and smaller available wafer sizes $\left(\sim 49 \mathrm{~cm}^{2}\right)$ have pushed the substitution of Si for traditional CdZnTe substrates. Although cheaper $\left(0.56 \$ / \mathrm{cm}^{2}\right)$ and larger in size $\left(5228 \mathrm{~cm}^{2}\right)$, the growth of high quality MCT on Si has been hindered by the high dislocation density induced by the large lattice mismatch (19.5\%) between Si and MCT, which will deteriorate detector performance parameters such as operability, sensitivity and uniformity among the focal plane array due to dead pixels [2]. Ex situ thermal cycle annealing (TCA) is one of the recent strategies explored for further reduction of MCT dislocation density. Although different etchants have been developed to delineate defects in MCT, no detailed TEM study has yet been reported on correlation of dislocations with different etch pit shapes. In this study, MBE-grown $\mathrm{Hg}_{0.8} \mathrm{Cd}_{0.2} \mathrm{Te}$ samples on $\mathrm{Si}(211)$ substrates were subjected to 4 annealing cycles between $250^{\circ} \mathrm{C}$ and $494^{\circ} \mathrm{C}$. Both as-grown and TCA samples were then defect-decorated using the Benson etchant. FIB milling with an FEI Nova200 was used to prepare cross-section specimens across different etch pit morphologies in $<0 \overline{1} 1>$ projections. A Philips-FEI CM-200 was used to image the samples at various orientations using conventional bright-field (BF) two-beam imaging for Burgers vector determination.

Figure 1(a) shows an SEM image of an as-grown MCT sample after etching. The total EPD was $\sim 1.1 \times 10^{8} \mathrm{~cm}^{-2}$ and the distribution of the pits in the as-grown sample was roughly $43 \%$ triangular, $29 \%$ skew, 26\% fish-eye, and 2\% others. Figures 1 (b-c) show BF XTEM micrographs of the region highlighted in Fig. 1(a) along bone-shape and triangular pits: the dislocation segment beneath the triangular pit goes in and out of contrast. Figures 2 (a-c) show a similar imaging approach for fish-eye shape pits. Complete $\boldsymbol{g . b}$ analysis determines that triangular pits for as-grown material are associated with a mixture of Frank partials and perfect dislocations and that fish-eye pits are associated with perfect dislocations. Comparison of SEM micrographs, Fig 3(a-b), of as-grown and TCA samples shows a drastic reduction, $(\sim 72 \%)$, in etch pit densities after TCA treatment. The total EPD after TCA was $\sim 3.1 \times 10^{7} \mathrm{~cm}^{-2}$ and the corresponding EPD for each type of pit was triangular pits $\sim 1.6 \times 10^{7} \mathrm{~cm}^{-2}$, skew pits $\sim 9.2 \times 10^{6} \mathrm{~cm}^{-2}$ and others $\sim 6.2 \times 10^{6} \mathrm{~cm}^{-2}$. A detailed analysis for these different pits in both asgrown and TCA samples has been conducted recently [3]. These observations connect the different types of etch pits with the dislocations that are present in as-grown and TCA MCT (211) films, and provide insight into the transformation and removal of dislocations during TCA.

References:

[1] A. Rogalski, J. Antoszewski, and L. Faraone, J. Appl. Phys. 105 (2009), 091101.

[2] P. Wijewarnasuriya et al, J. Electron. Mater. 39 (2010), 1110.

[3] M. Vaghayenegar et al, J. Electron. Mater. under review.

[4] This work was supported by Army Research Office Grant \#63749-EL. We gratefully acknowledge the use of facilities within the John M. Cowley Center for HREM at Arizona State University. 

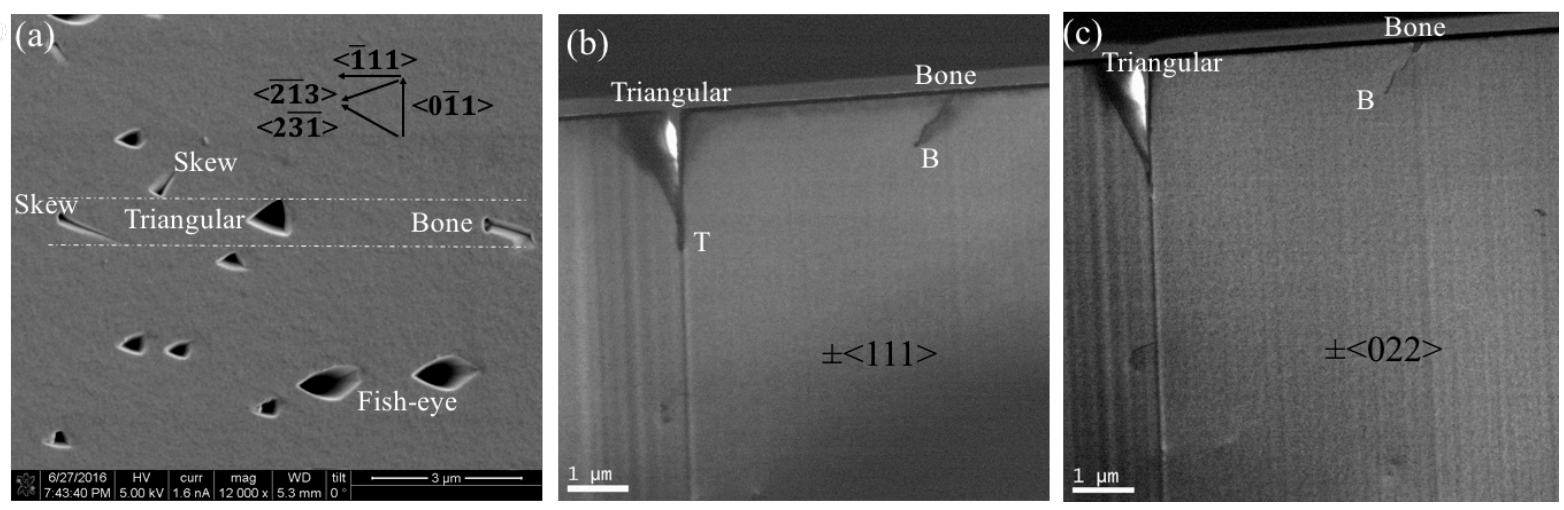

Figure 1. (a) SEM micrograph of as-grown MCT sample. $(b, c)$ XTEM BF images for triangular and boneshape pits at two different " $\boldsymbol{g}$ ". Segment marked "T" goes in and out of contrast.
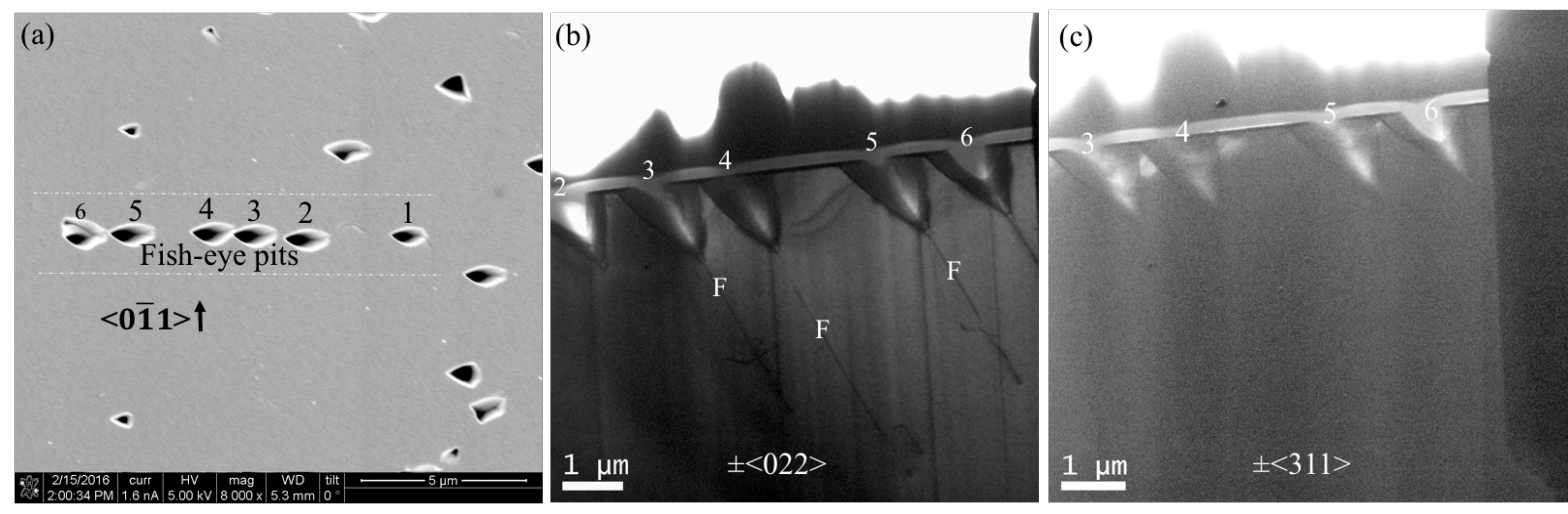

Figure 2. (a) SEM micrograph of a set of fish-eye shape pits in as-grown MCT material targeted for analysis; $(b, c)$ BF XTEM micrographs for these pits at two different " $g$ ". Segments marked "F" go in and out of contrast.
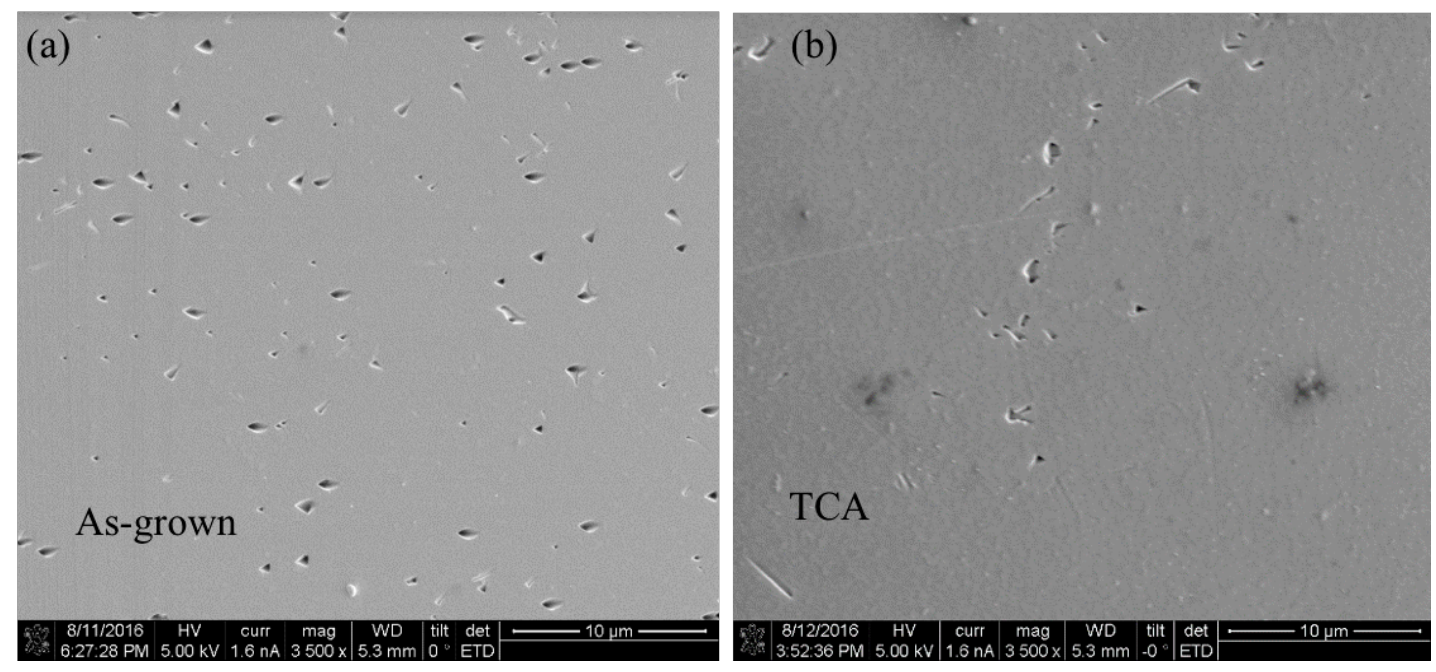

Figure 3. Comparison of SEM micrographs showing: (a) as-grown; and (b) TCA. Note the drastic reduction in density of etch pits after annealing. 